\title{
A THEOREM ON QUADRATIC FORMS AND ITS APPLICATION IN THE CALCULUS OF VARIATIONS*
}

\author{
BY \\ M. R. HESTENES AND E. J. McSHANE
}

1. Principal result. Let $P(z), Q_{1}(z), \cdots, Q_{r}(z)$ be quadratic forms in the real variables $z_{1}, \cdots, z_{m}$ with real coefficients having the following properties: the form $P(z)$ is positive at each point $(z) \neq(0)$ at which the forms $Q_{1}(z), \cdots, Q_{r}(z)$ vanish simultaneously; for every set of constants $S_{1}, \cdots, S_{r}$, not all zero, the quadratic form $S_{i} Q_{i}(z) \dagger$ is indefinite; for every linear subspace $L$ on which the quadratic forms $Q_{1}, \cdots, Q_{r}$ do not vanish simultaneously, save at $(z)=(0)$, there is a linear combination $S_{i} Q_{i}(z)$ of these forms which is positive definite on $L$. The principal result given in the present note is given in the following:

THEOREM 1. Under the above hypotheses there exists a set of constants $S_{1}, \cdots, S_{r}$ such that the quadratic form

$$
P(z, S)=P(z)+S_{i} Q_{i}(z) \quad(i=1, \cdots, r)
$$

is positive definite in the variables $z_{1}, \cdots, z_{m}$. The last two hypotheses may be dropped when $r=1$.

This theorem was proposed in a somewhat different form by Bliss in 1937 in a seminar on the calculus of variations. It is useful in sufficiency proofs for multiple integral problems. Proofs of the theorem for the case $r=1$ were given in the seminar by Albert, $\ddagger$ Reid, $\S$ McShane and Hestenes, each using a different method. The last two of these proofs have not been published. The proof of Theorem 1 here given is due to McShane and is an immediate extension of the one given by McShane for the case $r=1$. In the next section Theorem 1 will be applied to the case in which $(z)=\left(x_{1}, \cdots, x_{n}, y_{1}, \cdots, y_{n}\right)$ and the forms $Q_{1}, \cdots, Q_{r}$ are the two-rowed minors of the $n \times 2$-dimensional matrix $\left(x_{k} y_{k}\right)$. If $n=2$ then $r=1$ and Theorem 2 below is an immediate consequence of Theorem 1. If $n>2$ the further result described in Theorem 3 below is

* Presented separately to the Society, by McShane on April 8, 1939, and by M. R. Hestenes on December 29, 1939; received by the editors November 29, 1939.

$\dagger$ A repeated index denotes summation.

$\ddagger$ A. A. Albert, $A$ quadratic form problem in the calculus of variations, Bulletin of the American Mathematical Society, vol. 44 (1938), pp. 250-252.

$\S \mathrm{W}$. T. Reid, $A$ theorem on quadratic forms, Bulletin of the American Mathematical Society, vol. 44 (1938), pp. $437-440$. 
needed. This further result for the case $n=3$ has been established by McShane.* The proofs of Theorems 3, 4 and 5 here given are due to Hestenes. $\dagger$

The proof of Theorem 1 is based on two lemmas, the first of which is the following:

LeMma A. Let $m(S)$ be the minimum of the quadratic form $P(z, S)$ on the unit $(m-1)$-sphere $z_{k} z_{k}=1$. There exists a set of constants $\left(S_{0}\right)$ which maximizes the function $m(S)$.

For a set of constants $S_{1}, \cdots, S_{r}$ let $M(S)$ be the maximum of the quadratic form $-S_{i} Q_{i}$ on the unit sphere $z_{k} z_{k}=1$. We have $M(S)>0$ if $(S) \neq(0)$ since the quadratic form $-S_{i} Q_{i}$ is indefinite by hypothesis. Moreover $M(h S)=h M(S)$ for every positive number $h$. Let $M_{0}$ be the minimum of $M(S)$ on the set $S_{i} S_{i}=1$. Since this minimum is attained, we have $M_{0}>0$. Finally let $N$ be the maximum of $P(z)$ on the set $z_{k} z_{k}=1$. Then for every set of constants $(S)$ there is a point $(z)$ such that $z_{k} z_{k}=1,-S_{i} Q_{i}(z)=M(S) \geqq h M_{0}$, $S_{i} S_{i}=h^{2}$ and

$$
m(S) \leqq P(z, S) \leqq N-M(S) \leqq N-h M_{0} .
$$

It follows that there is a positive constant $h_{0}$ such that when $h>h_{0}$ we have $m(S)<m(0)$ for every set $(S)$ with $S_{i} S_{i}=h^{2}$. There is accordingly a set $\left(S_{0}\right)$ such that the relation $m(S) \leqq m\left(S_{0}\right)$ holds for every set $(S)$, as was to be proved.

Lemma B. Let $\left(S_{0}\right)$ be a set of constants which maximizes the function $m(S)$ and set $m_{0}=m\left(S_{0}\right)$. The set of points $(z)$ satisfying the equation $P\left(z, S_{0}\right)=m_{0} z_{k} z_{k}$ forms a linear space $L$. There is no quadratic form $S_{i} Q_{i}$ which is positive definite on $L$.

The set $L$ consists of all points $z$ at which the function $P\left(z, S_{0}\right)-m_{0} z_{k} z_{k}$ attains its minimum value 0 . Hence the partial derivatives of that function vanish on $L$, and therefore the equations

$$
P_{z_{k}}\left(z, S_{0}\right)=2 m_{0} z_{k}
$$

hold for all $z$ in $L$. Conversely, if we multiply equations (1) by $z_{k}$ and sum on $k$ we see that every point $z$ which satisfies (1) lies in $L$. Thus $L$ is the set of solutions of the linear equations (1), and is therefore linear. Suppose now that there exists a quadratic form $Q=S_{i} Q_{i}$ that is positive definite on $L$. Let $K$ be the unit sphere $z_{k} z_{k}=1$ and $L_{1}$ the set of points in $L$ on $K$. Choose $b>0$ such

* E. J. McShane, The condition of Legendre for double integral problems of the calculus of variations, Bulletin of the American Mathematical Society, abstract 45-5-209.

$\dagger$ M. R. Hestenes, $A$ theorem on quadratic forms and its application in the calculus of variations, Bulletin of the American Mathematical Society, abstract 46-1-83. 
that $Q(z)>b$ on $L_{1}$, and let $N$ be a neighborhood of $L_{1}$ relative to $K$ on which $Q(z)>b$. Let $m_{1}$ be the minimum of $P\left(z, S_{0}\right)$ on the closed set $K-N$. Then $m_{1}>m_{0}$. It follows that for a sufficiently small positive constant $h$ one will have

$$
P\left(z, S_{0}+h S\right)=P\left(z, S_{0}\right)+h Q>m_{0}
$$

on $K-N$. But $P\left(z, S_{0}+h S\right)>m_{0}+h b$ on $N$, and hence $m\left(S_{0}+h S\right)>m\left(S_{0}\right)$, contrary to our choice of the set $\left(S_{0}\right)$. This proves Lemma B.

In order to prove Theorem 1 we note that there is a point $(z) \neq(0)$ on the set $L$ described above at which the forms $Q_{1}, \cdots, Q_{r}$ vanish simultaneously. Otherwise by virtue of the last hypothesis made in Theorem 1 there would exist a quadratic form $S_{i} Q_{i}(z)$ which is positive definite on $L$, contrary to Lemma B. At this point $(z)$ we have accordingly $m_{0} z_{k} z_{k}=P\left(z, S_{0}\right)=P(z)>0$ in view of the first hypothesis of the theorem. It follows that $m_{0}>0$ and hence that $P\left(z, S_{0}\right)$ is positive definite. This proves the first statement in the theorem. The second statement is readily verified. It should be observed that the hypotheses of Theorem 1 imply that there is a point $(z) \neq(0)$ at which the forms $Q_{1}, \cdots, Q_{r}$ vanish simultaneously. Otherwise the last hypothesis would imply the existence of a positive definite form $S_{i} Q_{i}$, contrary to the second hypothesis.

The last sentence in Theorem 1 remains to be established. It is easy to see that if $r=1$ the last hypothesis in $\$ 1$ is automatically satisfied. Suppose then that $r=1$ and that $Q$ is not indefinite. Let $K$ be the sphere $z_{k} z_{k}=1$. If $Q_{1}$ is positive definite, the $\operatorname{sum} P(z, S)$ is positive on $K$, hence is a positive definite form, provided that $S_{1}$ is large. If $Q_{1}$ is negative definite, $P(z, S)$ is positive definite provided that $-S_{1}$ is large. If $Q_{1}$ is semi-definite, say positive, let $L$ be the set on which $Q_{1}$ vanishes. As in Lemma B, this is linear. On $L K$ the form $P(z)$ is positive; it then remains positive on a neighborhood $N$ of $L K$ relative to $K$. On $K-N$ the form $Q_{1}$ is positive. Choose $S_{1}$ large enough so that $P(z, S)$ is positive on $K-N$. On $N$ we have $P(z, S) \geqq P(z)>0$, so that $P(z, S)$ is positive on $K$, and is therefore a positive definite form.

In the proof of the first part of Theorem 1 made above we have established essentially the following more general result:

Corollary. Suppose the last two of the hypotheses made in Theorem 1 hold, and let $m$ be the minimum of the form $P(z)$ on the set of points $(z)$ satisfying the conditions $Q_{1}(z)=\cdots=Q_{r}(z)=0, z_{k} z_{k}=1$. There exists a set of constants $S_{i}$ such that the inequality $P(z, S) \geqq m z_{k} z_{k}$ holds for all points $(z)$.

2. A further result. Consider now the case in which the space of points $(z)$ described in the last section is of dimension $2 n$. For the purposes of this sec- 
tion it will be convenient to denote the $i$ th $(i \leqq n)$ and the $(n+i)$ th coordinates of $(z)$ by $x_{i}$ and $y_{i}$ respectively. Thus the points of our space will be denoted by the symbol $(x, y)$. To each point $(x, y)$ there is associated a $n \times 2$ dimensional matrix $\left(x_{i} y_{i}\right)$ whose $i$ th row is composed of the coordinates $x_{i}, y_{i}$ of the point $(x, y)$. This matrix will be used below to classify the points $(x, y)$ of our space. By a quadratic form in the variables $(x, y)$ will be meant an expression of the form

$$
R_{i k} x_{i} x_{k}+S_{i k} x_{i} y_{k}+T_{i k} y_{i} y_{k} .
$$

In particular the expression $S_{i k} x_{i} y_{k}$ is a quadratic form in the variables $(x, y)$. Finally by a linear space $L$ of points $(x, y)$ is meant a subspace such that if $(x, y)$ and $\left(x^{\prime}, y^{\prime}\right)$ belong to $L$ so also does the point $\left(a x+b x^{\prime}, a y+b y^{\prime}\right)$, where $a$ and $b$ are arbitrary real constants.

The results described in the last section will be used to prove the following:

THEOREM 2. Let $P(x, y)$ be a quadratic form in the $2 n$ real variables $x_{1}, \cdots, x_{n}, y_{1}, \cdots, y_{n}$ with real coefficients. Suppose that the inequality $P(x, y)>0$ holds whenever the $n \times 2$-dimensional matrix $\left(x_{i} y_{i}\right)$ has rank 1 . Then there exists an n-rowed skew-symmetric matrix $S=\left(S_{i k}\right)$ such that the quadratic form $P(x, y)+S_{i k} x_{i} y_{k}(i, k=1, \cdots, n)$ is positive definite.

To prove this result let $Q_{i k}(i<k)$ be the quadratic form $x_{i} y_{k}-x_{k} y_{i}$. A linear combination $S_{i k} Q_{i k}$ (summed with $i<k$ ) is easily seen to be equal to $S_{i k} x_{i} y_{k}$ (summed for all $\left.i, k\right)$ if we set $S_{i i}=0, S_{k i}=-S_{i k}(i<k)$. Thus we see that the theorem will be established if we show that the hypotheses of Theorem 1 with $Q_{i}$ replaced by $Q_{i k}$ are satisfied. The first hypothesis holds since the matrix $\left(x_{i} y_{i}\right) \neq(00)$ has rank 1 if and only if the forms $Q_{i k}$ vanish simultaneously. Moreover a linear combination $S_{i k} Q_{i k}$ with $S \neq 0$ is indefinite. Finally the last hypothesis of Theorem 1 holds by virtue of the following:

THEOREM 3. Let $L$ be a linear set of points $(x, y)$ such that the $n \times 2$-dimen. sional matrix $\left(x_{i} y_{i}\right)$ has rank 2 at each point $(x, y) \neq(0,0)$ on $L$. There exists a skew-symmetric matrix $S=\left(S_{i k}\right)$ such that the quadratic form $S_{i k} x_{i} y_{k}$ is positive definite on $L$.

Let $m$ be the dimension of $L$ and let $\left(X_{1 \alpha}, \cdots, X_{n \alpha}, Y_{1 \alpha}, \cdots, Y_{n \alpha}\right)$ $(\alpha=1, \cdots, m)$ be a basis for $L$, that is, a set of $m$ points $\left(X_{\alpha}, Y_{\alpha}\right)$ in $L$ such that the coordinates of each point $(x, y)$ in $L$ are expressible uniquely in the form

$$
x_{i}=X_{i \alpha} u_{\alpha}, \quad y_{i}=Y_{i \alpha} u_{\alpha} \quad(\alpha=1, \cdots, m) .
$$

Since the matrix $\left(x_{i} y_{i}\right)$ has rank 2 at each point $(x, y) \neq(0,0)$ on $L$, the matrix 
$\lambda X+\mu Y$, where $X=\left(X_{i \alpha}\right)$ and $Y=\left(Y_{i \alpha}\right)$, has rank $m$ for every pair of real numbers $\lambda, \mu$, not both zero. Conversely for every pair of matrices $X, Y$, having $\lambda X+\mu Y$ of rank $m$ when $(\lambda, \mu) \neq(0,0)$, the corresponding linear space $L$ defined by equations (2) is such that the matrix $\left(x_{i} y_{i}\right)$ has rank 2 at each point $(x, y) \neq(0,0)$ on $L$. Moreover by the use of equations (2) it is seen that $S_{i k} x_{i} y_{k}=R_{\alpha \beta} u_{\alpha} u_{\beta}$, where $R=X^{\prime} S Y$ and $X^{\prime}$ is the transpose of $X$. Here and elsewhere it is understood that the symbol for an element of a matrix is obtained by adding a pair of subscripts to the symbol for the matrix. Theorem 3 is accordingly equivalent to the following theorem on matrices:

THEOREM 4. Let $X, Y$ be two $n \times m$-dimensional matrices such that the matrix $\lambda X+\mu Y$ has rank $m$ for every pair of real numbers $\lambda, \mu$, not both zero. There exists an n-rowed skew-symmetric matrix $S$ such that the matrix $R=X^{\prime} S Y$ is positive definite, that is, the quadratic form $R_{\alpha \beta} u_{\alpha} u_{\beta}(\alpha, \beta=1, \cdots, m)$ is positive definite. Here $X^{\prime}$ is the transpose of $X$. The matrix $R$ in general will not be symmetric.

In order to prove Theorem 4 we first observe that in the proof of the theorem we may replace the matrices $X, Y$ by $X_{1}=A X B, Y_{1}=A Y B$, where $A$ and $B$ are arbitrary nonsingular matrices of dimensions $n$ and $m$ respectively. For, the matrix $\lambda X_{1}+\mu Y_{1}$ has rank $m$ when $(\lambda, \mu) \neq(0,0)$. Moreover, suppose there exists a skew-symmetric matrix $S_{1}$ such that the matrix $X_{1}^{\prime} S_{1} Y_{1}$ is positive definite. Let $S$ be the skew-symmetric matrix $A^{\prime} S_{1} A$, and set $R=X^{\prime} S Y$. Then the matrix $B^{\prime} R B=X_{1}^{\prime} S_{1} Y_{1}$ is positive definite. It follows readily that $R$ is positive definite and hence that the matrices $X, Y$ can be replaced by $X_{1}, Y_{1}$ respectively. In fact $X, Y$ can be replaced by matrices of the form

$$
X=\left(\begin{array}{l}
I \\
O
\end{array}\right), \quad Y=\left(\begin{array}{l}
C \\
D
\end{array}\right),
$$

where $I$ is the $m$-rowed identity matrix, $C$ is an $m$-rowed matrix and $O$ is the zero matrix. Suppose that $X, Y$ are of this form. Then the condition that the matrix $\lambda X+\mu Y$ be of rank $m$ for all real numbers $\lambda$, $\mu$, not both zero, is equivalent to the condition that the matrix

$$
\left(\begin{array}{c}
\lambda I-C \\
-D
\end{array}\right)
$$

be of rank $m$ for all real values of $\lambda$. Moreover the equation $A X B=X$ holds for two nonsingular matrices $A$ and $B$ if and only if $A$ is of the form

$$
A=\left(\begin{array}{cc}
B^{-1} & B^{-1} E \\
O & F
\end{array}\right)
$$


When $A$ is of this form we have

$$
A Y B=\left(\begin{array}{c}
B^{-1}(C+E D) B \\
F D B
\end{array}\right) .
$$

In the sequel we shall assume that the matrices $X, Y, A$ are of the forms (3) and (5). It will be understood that if $n=m$ the matrices $O, D, E, F$ do not appear in these matrices. Theorem 4 will be established by replacing $Y$ by a matrix $A Y B$ having special properties. This will be done with the help of three lemmas, the first of which is the following:

Lemma 1. Let $\phi_{1}(\lambda), \cdots, \phi_{k}(\lambda)$ be the elementary divisors of the matrix $\lambda I-C$ and let $\epsilon$ be an arbitrary constant different from zero. With an elementary divisor of the form $\phi_{i}(\lambda)=\left[\left(\lambda-\alpha_{i}\right)^{2}+\beta_{i}^{2}\right]^{m_{i}}\left(\beta_{i} \neq 0\right)$ associate the $2 m_{i}$-rowed matrix

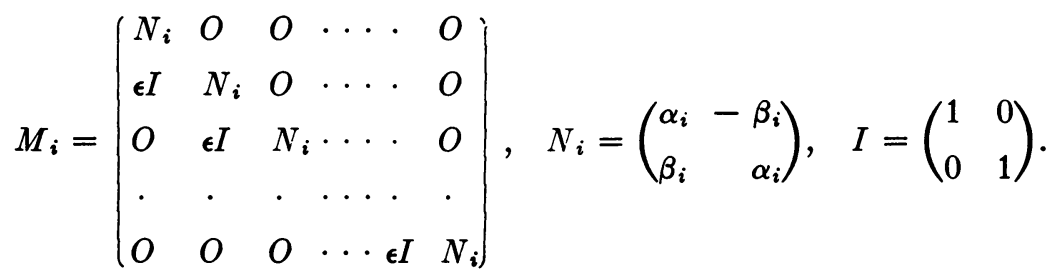

With an elementary divisor of the form $\phi_{j}(\lambda)=\left(\lambda-\alpha_{j}\right)^{m_{j}}$ associate the matrix

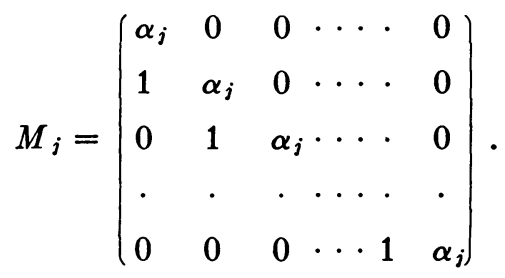

There exists a nonsingular $m$-rowed matrix $B$ such that $B^{-1} C B=C_{1}$, where

$$
C_{1}=\left(\begin{array}{lllll}
M_{1} & O & O & \cdots & O \\
O & M_{2} & O & \cdots & O \\
O & O & M_{3} & \cdots & O \\
\cdot & \cdot & \cdot & \cdots & \cdot \\
O & O & O & \cdots & M_{k}
\end{array}\right)
$$

For it is clear from the special forms (7) and (8) that $\phi_{i}(\lambda)=\left|\lambda I-M_{i}\right|$. If $M_{i}$ is of the form (8), the $\left(m_{i}-1\right)$-rowed minor obtained by deleting the first row and last column has determinant unity. Hence $\phi_{i}(\lambda)$ is the only nontrivial invariant factor of $\lambda I-M_{i}$ in this case. Suppose next that $M_{i}$ is of the 
form (7) and let $\phi(\lambda)$ be the determinant of the minor of $\lambda I-M_{i}$ obtained by deleting the first row and the last column. The values $\phi\left(\alpha_{i}+i \beta_{i}\right), \phi\left(\alpha_{i}-i \beta_{i}\right)$ are different from zero since $\epsilon \neq 0$, as one readily verifies. It follows that $\phi(\lambda)$ and $\phi_{i}(\lambda)$ have no common factors and hence that $\phi_{i}(\lambda)$ is the only non-trivial invariant factor of $\lambda I-M_{i}$ when $M_{i}$ is of the form (7). The matrices $\lambda I-C_{1}$ and $\lambda I-C$ accordingly have the same elementary divisors and hence the same invariant factors. The matrix $C_{1}$ is therefore similar to $C$, that is, $C_{1}$ is of the form $B^{-1} C B$, where $B$ is nonsingular. $\dagger$

LEмма 2. If the equation $|\lambda I-C|=0$ has no real roots, there exists a matrix $C^{*}$ similar to $C$ and a skew-symmetric matrix $S$ such that the matrix $S C^{*}$ is positive definite. The matrix $S C^{*}$ is not in general symmetric.

For in this case the diagonal blocks in (9) are of the form (7). Consider $C_{1}$ as a function $C_{1}(\alpha, \beta, \epsilon)$ of the values $\alpha_{i}, \beta_{i}, \epsilon$ described in Lemma 1 . Let $R(\epsilon)=C_{1}(0, \beta, \epsilon), S=-R(0)$ and $T=C_{1}(\alpha, 0,0)$. Then $S$ and $S T$ are skewsymmetric, $S$ is nonsingular and $C_{1}=R(\epsilon)+T$. Since $S T$ is skew-symmetric one has $u_{\alpha} S_{\alpha \beta} T_{\beta \gamma} u_{\gamma}=0(\alpha, \beta, \gamma=1, \cdots, m)$ and

$$
u_{\alpha} S_{\alpha \beta}\left(R_{\beta \gamma}+T_{\beta \gamma}\right) u_{\gamma}=u_{\alpha} S_{\alpha \beta} R_{\beta \gamma} u_{\gamma}
$$

for arbitrary values of $(u)$. Since $R(0)=-S$ and $S$ is nonsingular, the last quadratic form is positive definite when $\epsilon=0$ and hence for a value $\epsilon^{\prime} \neq 0$. The matrices $S$ and $C^{*}=R\left(\epsilon^{\prime}\right)+T$ have the properties described in the lemma.

Lemma 3. Let $C, D$ be the matrices appearing in the matrix (4) and suppose the matrix (4) has rank $m$ for all real values of $\lambda$. There exists an $m \times(n-m)-$ dimensional matrix $E$ such that the equation

$$
|\lambda I-C-E D|=0
$$

has no real roots if the dimension $m$ of $C$ is even and a single real root if $m$ is odd.

Let us begin by disposing of the case $m=n$. If $m=n$, the conclusion of Lemma 3 is that $|\lambda I-C| \neq 0$ for all real $\lambda$. This is an immediate consequence of the hypothesis that matrix (4) has rank $m=n$ for all real $\lambda$. Incidentally, if $m=n$, both $m$ and $n$ must be even; otherwise the equation $|\lambda I-C|=0$ would be of odd degree, and would therefore have a real root.

Suppose then that $m<n$. We shall consider first the case in which $m=2$ and $C, D$ are of the form

$$
C=\left(\begin{array}{ll}
a & 0 \\
c & b
\end{array}\right), \quad D=\left(\begin{array}{ll}
1 & 0 \\
0 & 1
\end{array}\right) \text { or } \quad D=\left(\begin{array}{ll}
d & e
\end{array}\right) .
$$

† A. A. Albert, Modern Higher Algebra, University of Chicago Press, 1937, pp. 84-85. 
If $D$ is the two-rowed identity matrix, let

$$
G=\left(\begin{array}{rr}
0 & -1 \\
1 & 0
\end{array}\right), \quad E=G-C .
$$

Then the equation (10) takes the form $\lambda^{2}+1=0$ and has no real roots. Suppose next that $D=(d e)$. We may suppose that $b=0$ since this can be brought about by replacing $\lambda$ by $\lambda+b$. Since the matrix (4) has rank 2 for $\lambda=0$ and $\lambda=a$, we have $e \neq 0$ and $a d+e c \neq 0$. Choose numbers $\alpha, \beta$ such that

$$
\alpha c e-\beta a e=-1, \quad \alpha d+\beta e+a=0 .
$$

Then equation (10) with $E^{\prime}=(\alpha \beta)$ as the transpose of $E$ reduces to $\lambda^{2}+1=0$. The lemma is accordingly true for the matrices (11).

To prove the lemma as stated, let $h$ be the number of real roots of the equation $|\lambda I-C|=0$, each root counted a number of times equal to its multiplicity. We may suppose that $h \geqq 2$. It is sufficient to show that the matrix $E$ can be chosen so that the equation (10) has exactly $h-2$ real roots. In view of equation (6) it is sufficient to prove this result when $C, D$ are replaced respectively by matrices of the form $C_{1}=B^{-1} C B, D_{1}=F D B$, where $B$ and $F$ are nonsingular. By virtue of Lemma 1 we may select $B$ so that $C_{1}$ is given by (9). In fact $B$ may be chosen so that the matrices (8) corresponding to real roots of $|\lambda I-C|=0$ have higher subscripts than the matrices $(7)$ corresponding to roots that are not real. It follows that, after a suitable choice of the matrix $F$ in $D_{1}$, one has

$$
C_{1}=\left(\begin{array}{ll}
M & O \\
N & C_{2}
\end{array}\right), \quad D_{1}=\left(\begin{array}{ll}
U & O \\
V & D_{2}
\end{array}\right),
$$

where the matrices $C_{2}, D_{2}$ are the matrices $C, D$ in (11). The matrix (4) with $C=C_{2}, D=D_{2}$ has rank 2 , since otherwise the corresponding matrix with $C=C_{1}, D=D_{1}$ could not have rank $m$. It follows that there exists a matrix $E_{2}$ such that the equation (10) with $C=C_{2}, D=D_{2}, E=E_{2}$ has no real roots. Choosing

$$
E=\left(\begin{array}{ll}
O & O \\
O & E_{2}
\end{array}\right)
$$

the equation (10) with $C=C_{1}, D=D_{1}$ reduces to the product

$$
\left|\lambda I_{m-2}-M\right| \cdot\left|\lambda I_{2}-C_{2}-E_{2} D_{2}\right|=0
$$

where the subscript on $I$ denotes its dimension. This equation has $h-2$ real roots and the proof of Lemma 3 is complete.

We are now in position to prove Theorem 4. As was seen above we can assume that the matrices $X, Y$ are of the form (3). Since we can replace $Y$ 
by the matrix (6) we can suppose, by virtue of Lemma 3, that the equation $|\lambda I-C|=0$ has no real roots if the dimension $m$ of $C$ is even and one real root if $m$ is odd. Consider first the case in which $m$ is even. Then by Lemma 2 there is a nonsingular matrix $B$ and a skew-symmetric matrix $S$ such that the matrix $S C^{*}$ with $C^{*}=B^{-1} C B$ is positive definite. Replace $Y$ by $A Y B$, where $A$ is defined by equation (5) with $E=0$ and $F=I$. Then $C$ is replaced by $C^{*}$ so that the matrix $S C$ is now positive definite. Let $S_{1}$ be the $n$-rowed skew-symmetric matrix

$$
\left(\begin{array}{ll}
S & O \\
O & O
\end{array}\right)
$$

The product $X^{\prime} S_{1} Y$ is then equal to $S C$ and is accordingly positive definite. This proves Theorem 4 for the case in which $m$ is even.

The case in which $m$ is odd will be reduced to the case in which $m$ is even. As was seen above we can assume that the equation $|\beta I-C|=0$ has only one real root $\lambda=a$. Choose nonsingular matrices $B, F$ such that the matrices $C_{1}=B^{\prime} C B, D_{1}=F D B$ are of the form

$$
C_{1}=\left(\begin{array}{cc}
M & O \\
O & a
\end{array}\right), \quad D_{1}=\left(\begin{array}{cc}
U & b \\
V & O
\end{array}\right) \quad(b \neq 0) .
$$

This choice is possible by virtue of Lemma 1 with $M_{k}=(a)$ and the fact that the matrix (4) has rank $m$ when $\lambda=a$. We can suppose that $C=C_{1}, D=D_{1}$, since this result can be brought about by replacing $Y$ by $A Y B$, where $A$ is given by (5) with $E=O$. Clearly $m<n$ since $D$ has at least one row. Let $X_{1}, Y_{1}$ be the matrices

$$
X_{1}=\left(\begin{array}{lll}
I & O & 0 \\
O & 1 & 0 \\
O & 0 & 1 \\
O & O & O
\end{array}\right), \quad Y_{1}=\left(\begin{array}{ccr}
M & O & O \\
O & a & -b \\
U & b & a \\
V & O & O
\end{array}\right)
$$

obtained by adding a suitable column to each of the matrices $X$ and $Y$. Since the equations $|\lambda I-M|=0,(\lambda-a)^{2}+b^{2}=0$ have no real roots, the matrix $\lambda X_{1}-Y_{1}$ corresponding to (4) has rank $m+1$ for all real values of $\lambda$. As was seen in the last paragraph, there exists, since $m+1$ is even, a skew-symmetric matrix $S$ such that the matrix $X_{1}^{\prime} S Y_{1}$ is positive definite. The matrix $X^{\prime} S Y$, being a principal minor of $X_{1}^{\prime} S Y_{1}$, is also positive definite. This completes the proof of Theorem 4 and hence also of Theorem 3 .

Incidentally, by an argument similar to that just made, it can be shown that if $L$ is a maximal linear space having the properties described in Theorem 3 then its dimension is $n$ if $n$ is even and $n-1$ if $n$ is odd. 
3. Application to the calculus of variations. Consider the problem of minimizing the double integral

$$
I=\iint_{A} f\left(x, y, z_{1}, \cdots, z_{n}, p_{1}, \cdots, p_{n}, q_{1}, \cdots, q_{n}\right) d x d y,
$$

where $p_{i}=\partial z_{i} / \partial x, q_{i}=\partial z_{i} / \partial y$, in a class of subspaces

$$
z_{i}(x, y) \quad((x, y) \text { on } A+B ; i=1, \cdots, n)
$$

having a common boundary, where $B$ is the boundary of $A$. The integrand $f(x, y, z, p, q)$ is assumed to be continuous and to have continuous first and second partial derivatives on a region $R$ of points $(x, y, z, p, q)$. The subspaces (12) are assumed to be continuous, to have continuous second partial derivatives and to have their elements $(x, y, z, p, q)$ in $R$. The boundary $B$ of $A$ is supposed to be a simply closed continuous curve having a piecewise continuously turning tangent. Weaker differentiability assumptions could be made.

Lemma 4. Let $S_{i k}(x, y)=-S_{k i}(x, y)(i, k=1, \cdots, n)$ be arbitrary continuous functions having continuous first and second derivatives in a neighborhood of the set $A+B$. Then the integral

$$
\begin{aligned}
J & =\iint_{A} g(x, y, z, p, q) d x d y \\
& \equiv \iint_{A}(1 / 2)\left\{p_{i} \frac{\partial}{\partial y}\left(s_{i k} z_{k}\right)-q_{i} \frac{\partial}{\partial x}\left(s_{i k} z_{k}\right)\right\} d x d y
\end{aligned}
$$

has the same value for all subspaces (12) here considered.

For by virtue of Green's theorem the value of $2 J$ is given by the formula

$$
2 J=\iint_{A}\left\{\frac{\partial}{\partial y}\left(S_{i k} p_{i} z_{k}\right)-\frac{\partial}{\partial x}\left(S_{i k} q_{i} z_{k}\right)\right\} d x d y=\int_{B} z_{k} S_{k i} d z_{i},
$$

and hence is completely determined by the common boundary of the subspaces (12).

Let $E$ be a minimizing subspace. Then $E$ must satisfy the condition of Legendre, ${ }^{*}$ that is, at each element $(x, y, z, p, q)$ on $E$ the inequality

$$
P(\xi, \eta)=f_{p_{i} p_{k}} \xi_{i} \xi_{k}+2 f_{p_{i} q_{k}} \xi_{i} \eta_{k}+f_{q_{i} q_{k}} \eta_{i} \eta_{k} \geqq 0
$$

must hold for every set $(\xi, \eta)$ whose $n \times 2$-dimensional matrix has rank 1 . If

* Graves, The Weierstrass condition for multiple integral variation problems, Duke Mathematical Journal, vol. 5 (1939), pp. 656-660. Graves proves only the Weierstrass condition. The condition of Legendre is a well known consequence of that of Weierstrass. 
the condition (14) with the equality excluded holds on $E$ for every set $(\xi, \eta)$ whose matrix $\left(\xi_{i} \eta_{i}\right)$ has rank 1 , then $E$ will be said to satisfy the strengthened condition of Legendre. It should be emphasized that in this strengthened condition of Legendre we require that the inequality $P(\xi, \eta)>0$ hold only for sets $(\xi, \eta)$ whose $n \times 2$-dimensional matrix $\left(\xi_{i} \eta_{i}\right)$ has rank 1 and not for all sets $(\xi, \eta) \neq(0,0)$. We have the following:

THEOREM 5. Let E be a subspace (12) satisfying the strengthened condition of Legendre. There exists an invariant integral of the form (13) such that for the function $F=f+g$ the inequality

$$
F_{p_{i} p_{k}} \xi_{i} \xi_{k}+2 F_{p_{i} q_{k}} \xi_{i} \eta_{k}+F_{q_{i} q_{k}} \eta_{i} \eta_{k}>0
$$

holds at each element $(x, y, z, p, q)$ on $E$ for every $\operatorname{set}(\xi, \eta) \neq(0,0)$.

For by Theorem 2 there exists for each point on $E$ a skew-symmetric matrix $\left(S_{i k}\right)$ such that the quadratic form $P(\xi, \eta)+S_{i k} \xi_{i} \eta_{k}$ is positive definite at this point and hence in a neighborhood of this point. It follows readily that the set $A+B$ can be covered by a finite number of circles $C_{1}, \cdots, C_{m}$ having a common radius $r$ such that to the circle $C_{\alpha}{ }_{\alpha}$ of radius $2 r$ concentric with the circle $C_{\alpha}$ there is associated a skew-symmetric matrix $\left(S_{\imath k}^{\alpha}\right)$ of constant elements such that the form $P_{\alpha}(\xi, \eta)=P(\xi, \eta)+S_{i k}^{\alpha} \xi_{i} \eta_{k}$ is positive definite at each point on $E$ whose projection in the $x y$-plane is in $C_{\alpha}{ }^{\prime}$. We propose now to construct a set of functions $S_{i k}(x, y)=-S_{k i}(x, y)$ having continuous second derivatives on a neighborhood of $A+B$ and such that the form $P(\xi, \eta)+S_{i k}(x, y) \xi_{i} \eta_{k}$ is positive definite on $E$. To this end let $h(t)$ be a function having continuous second derivatives and such that $h(t)=0$ when $t \leqq r$, $h(t)=1$ when $t \geqq 2 r$, and $0<h(t)<1$ when $r<t<2 r$. Let $\left(x_{\alpha}, y_{\alpha}\right)$ be the center of the circle $C_{\alpha}$, and let $d_{\alpha}(x, y)$ be the distance between the points $(x, y)$ and $\left(x_{\alpha}, y_{\alpha}\right)$. Set $h_{\alpha}(x, y)=h\left[d_{\alpha}(x, y)\right]$. It should be observed that the product $h_{1} h_{2} \cdots h_{m}$ is identically zero on the set $A+B$. This follows because $h_{\alpha} \equiv 0$ on $C_{\alpha}$ and the circles $C_{1}, \cdots, C_{m}$ cover $A+B$. Denote by $S_{i k}(x, y)$ the function

$$
\left(1-h_{1}\right) S_{i k}^{1}+h_{1}\left(1-h_{2}\right) S_{i k}^{2}+\cdots+h_{1} h_{2} \cdots h_{m-1}\left(1-h_{m}\right) S_{i k}^{m},
$$

where $S_{\mathfrak{k}}^{\alpha}(\alpha=1, \cdots, m)$ are the constants described above. The quadratic form $P(\xi, \eta)+S_{i k}(x, y) \xi_{i} \eta_{k}$ determined by these functions is easily seen to be identical with the sum

$$
\left(1-h_{1}\right) P_{1}(\xi, \eta)+h_{1}\left(1-h_{2}\right) P_{2}(\xi, \eta)+\cdots+h_{1} h_{2} \cdots h_{m-1}\left(1-h_{m}\right) P_{m}(\xi, \eta),
$$

where $P_{\alpha}(\xi, \eta)$ is the quadratic form associated with the circle $C^{\prime}$. Each term in this sum is positive or zero, since for each integer $\alpha$ the product 
$\left(1-h_{\alpha}\right) P_{\alpha}(\xi, \eta)$ is positive definite at points on $E$ corresponding to points in $C_{2}^{\prime}$ and identically zero elsewhere. Moreover the terms do not vanish simultaneously when $(\xi, \eta) \neq(0,0)$. The quadratic form $P(\xi, \eta)+S_{i k} \xi_{i} \eta_{k}$ is accordingly positive definite on $E$.

We now use these functions $S_{i k}$ to define the function $g$ of equation (13). From the definition of $g$ we at once obtain

$$
g(x, y, z, p, q)=(1 / 2)\left[\left(\partial S_{i k} / \partial y\right) p_{i} z_{k}+\left(\partial S_{k i} / \partial x\right) q_{i} z_{k}\right]+S_{i k} p_{i} q_{k} .
$$

This shows that the Legendre quadratic form (15) for $g$ is $S_{i k} \xi_{i} \eta_{k}$. Hence if $F=f+g$ the Legendre form (15) for $F$ is exactly the positive definite form $P(\xi, \eta)+S_{i k} \xi_{i} \eta_{k}$. This establishes Theorem 5 .

The University of Chicago, Chicago, Ill.,

THE UNIVERSITY OF VIRGINIA, Chardottesville, Va. 